\title{
Hollow plasma channel for positron plasma wakefield acceleration
}

\author{
W. D. Kimura* \\ STI Optronics, Inc., 2755 Northup Way, Bellevue, Washington 98004, USA \\ H. M. Milchberg \\ Institute for Physical Science and Technology, University of Maryland, College Park, Maryland 20742, USA \\ P. Muggli and X. Li \\ University of Southern California, Los Angeles, California 90089, USA \\ W. B. Mori \\ University of California at Los Angeles, Los Angeles, California 90024, USA
}

(Received 9 September 2010; published 18 April 2011)

\begin{abstract}
Plasma wakefield acceleration (PWFA) has demonstrated the ability to produce very high gradients to accelerate electrons and positrons. In PWFA, a drive bunch of charged particles passes through a uniform plasma, thereby generating a wakefield that accelerates a witness bunch traveling behind the drive bunch. This process works well for electrons, but much less so for positrons due to the positive charge attracting rather than repealing the plasma electrons, which leads to reduced acceleration gradient, halo formation, and emittance growth. This problem can be alleviated by having the positron beam travel through a hollow plasma channel. Presented are modeling results for producing 10-100 cm long hollow plasma channels suitable for positron PWFA. These channels are created utilizing laser-induced gas breakdown in hydrogen gas. The results show that hollow channels with plasma densities of order $10^{16} \mathrm{~cm}^{-3}$ and inner channel radii of order $20 \mu \mathrm{m}$ are possible using currently available terawatt-level lasers. At these densities and radii, preliminary positron PWFA modeling indicates that longitudinal electric fields on axis can exceed $3 \mathrm{GV} / \mathrm{m}$.
\end{abstract}

DOI: 10.1103/PhysRevSTAB.14.041301

PACS numbers: 41.75.Lx, 52.50.Jm, 41.75.Ht, 52.65.Kj

\section{INTRODUCTION}

Plasma-based particle accelerators have made tremendous progress in recent years. One such scheme is a particle beam-driven accelerator known as a plasma wakefield accelerator (PWFA) [1]. In a proof-of-principle PWFA experiment [2], the energy of incoming $42 \mathrm{GeV}$ electrons was doubled over a plasma length of $\approx 85 \mathrm{~cm}$, corresponding to a gradient of nearly $50 \mathrm{GeV} / \mathrm{m}$.

In PWFA, a relativistic charged particle "drive" bunch (DB) travels through a uniform plasma and generates a wakefield traveling at near the speed of light. A "witness" bunch (WB) that follows the drive bunch can be phased with the wakefield such that the wakefield accelerates the bunch. This process works for both electrons and positrons; however, as explained shortly, certain problems arise for the positrons.

Future electron/positron machines, such as the International Linear Collider (ILC) or the Compact

\footnotetext{
*wkimura@stioptronics.com
}

Published by American Physical Society under the terms of the Creative Commons Attribution 3.0 License. Further distribution of this work must maintain attribution to the author(s) and the published article's title, journal citation, and DOI.
Linear Collider (CLIC) are designed to reach electron and positron beam energies between $125 \mathrm{GeV}$ and $1.5 \mathrm{TeV}$. However, these rf accelerators produce acceleration gradients of between 31.5 (ILC) and $150 \mathrm{MeV} / \mathrm{m}$ (CLIC, $30 \mathrm{GHz}$ ). These machines must therefore be tens of kilometers long to reach the desired energies. PWFA is one of the candidates that would increase the average gradient of such machines and therefore significantly reduce their size and cost. A PWFA scheme called the plasma afterburner [3] has been proposed to double the energy of a linear collider without doubling its length, thereby approximately halving the collider length for a given center-of-mass energy.

The physics of the interaction of electron beams with plasmas is different from that of positron beams with plasmas. In both cases, the plasma electrons are the mobile species. With an electron beam driver, a favorable situation is reached when the beam density is larger than the plasma density. In this nonlinear or blowout regime [4], the space charge field of the drive bunch expels all the plasma electrons from the bunch volume. The wakefields in the plasma ion column are such that the transverse focusing force is substantial, it increases linearly with radius (ideal focusing lens), and it is constant along the core of a single bunch or along the witness bunch. This means the electron 
beam can propagate in the plasma over long distances without significant emittance growth [5]. The accelerating field is also 3 to 5 times larger than in the linear regime and it is also independent of radius. This combination of strong, aberration-free focusing and large accelerating gradient explains the high energy gain observed with electrons.

In the case of a positron beam driver, the plasma electrons are attracted toward (rather than expelled from) the bunch and stream through it [6]. As a result, the focusing force is radially nonlinear, and varies along the bunch. Furthermore, the accelerating gradient is less than with an electron beam driver because the plasma electrons do not return as an ensemble into a small volume on the beam propagation axis. Fortunately, numerical simulations indicate that the acceleration gradient created by a positron drive bunch can be increased by using a hollow plasma channel rather than a homogeneous, uniform plasma [6]. The hollow channel diameter is chosen so that the plasma electrons rush together as a group towards the beam axis, thereby leading to a wake configuration more similar to that driven by an electron bunch. In addition, since the positron bunch propagates in nonionized gas or, ideally, vacuum, its emittance will also be better preserved upon acceleration.

In the case of an electron beam driver, the plasma electrons are expelled to a distance of approximately one plasma collisionless skin depth $\left(c / \omega_{p}\right)$, where $c$ is the speed of light and $\omega_{p}$ is the plasma frequency. (Recall $\omega_{p} \propto n_{e}^{1 / 2}$, where $n_{e}$ is the plasma density.) Preliminary simulations with a hollow channel consisting of a stepfunction gas/plasma boundary indicate that a channel with a radius of the order of $c / \omega_{p}$ maximizes the wake amplitude driven by a positron bunch [6].

Initial experiments [7] of guiding positron beams in a hollow plasma channel gave encouraging results, but the particular masking method used to create the hollow channel produced a channel that was partially filled. A better method for generating a hollow plasma channel is to use laser-induced gas breakdown in which a laser beam with a high-order Bessel-beam transverse intensity profile is generated by sending the beam through a phase plate (kinoform) and axicon lens. This is illustrated schematically in Fig. 1. If the laser intensity on axis is sufficient, it can ionize the gas along the line focus. The high-order

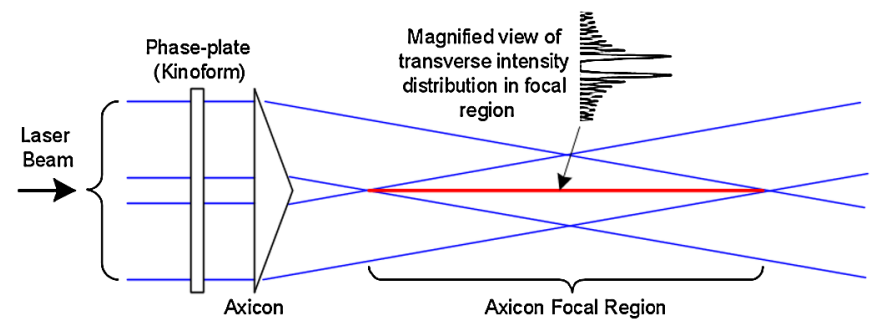

FIG. 1. Schematic of focusing a laser beam with an axicon and phase plate.
Bessel-beam intensity distribution within the focal region, as illustrated in Fig. 1, consists of a ring of high intensity centered on axis with a minimum in the center. Gas breakdown occurs primarily at the ring, resulting in the formation of a hollow or tubular plasma channel along the axicon focal region.

Fan et al. [8] experimentally demonstrated this method where they created a $0.8 \mathrm{~cm}$ long hollow plasma channel in 700 Torr of argon. Using a kinoform design developed by Andreev et al. [9,10], Fan et al. transformed their $500 \mathrm{~mJ}$, 100 ps Nd:YAG laser beam into a fifth-order, $J_{5}$, Bessel intensity profile. This produced a channel where the radius of the first maximum of the Bessel-beam focus was $\sim 4 \mu \mathrm{m}$ and the peak plasma density in the walls of the channel was $\sim 3 \times 10^{19} \mathrm{~cm}^{-3}$.

This experiment proved the viability of the technique; however, a plasma density of $10^{19} \mathrm{~cm}^{-3}$ would correspond to a plasma wavelength, $\lambda_{p} \propto n_{e}^{-1 / 2}$, that is too short for available positron bunch lengths. Nonetheless, a high $n_{e}$ is desirable because multi- $\mathrm{GeV} / \mathrm{m}$ energy gains are then possible [1,11]. Positron bunch lengths $<100 \mu \mathrm{m}$ are feasible [11]; hence, a channel wall density of $6 \times 10^{16} \mathrm{~cm}^{-3}$, corresponding to $\lambda_{p}$ slightly longer than $100 \mu \mathrm{m}$, was selected as the target value for our modeling effort. Then based upon the aforementioned $c / \omega_{p}$ criterion, at this density the inner radius of the channel should be $\sim 22 \mu \mathrm{m}$.

With these requirements in mind, we performed a modeling analysis to determine the conditions necessary to create 10-100 cm long hollow plasma channels in hydrogen gas with a channel radius of $\sim 20 \mu \mathrm{m}$ and a wall density of $6 \times 10^{16} \mathrm{~cm}^{-3}$. Of particular interest are the laser beam characteristics needed to produce these channels and the question of whether existing lasers have the needed capability. As we will show, the answer to this question is yes.

The next section reviews preliminary positron PWFA analysis and simulation work done to verify the target parameter values for the hollow plasma channel. Sections III and IV describe the model used to simulate formation of the hollow plasma channel and summarizes the results, respectively. Section IV ends with a discussion and conclusion. The Appendix contains a first-order analysis of the intensity distribution along the axicon focal region.

\section{PRELIMINARY POSITRON PWFA ANALYSIS AND SIMULATIONS}

\section{A. Ionization of hydrogen gas}

The key hollow plasma channel parameters are the radius $r_{\mathrm{ch}}$ of the hollow channel, the plasma density at the channel walls $n_{e}$, the channel length $L$, and the gas composition. The reasons for selecting the values for $r_{\mathrm{ch}}$ and $n_{e}$ were already given, and the goal is to make $L$ as 


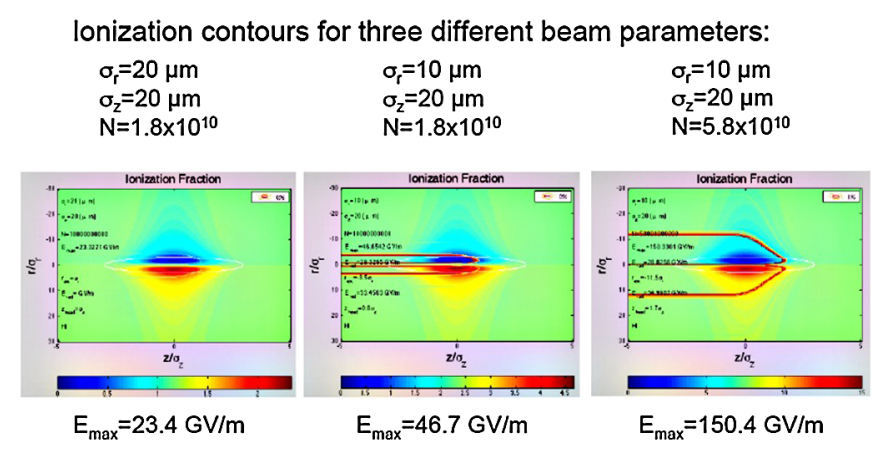

FIG. 2. Simulation of hydrogen ionization by positron beam. In the left figure, the field is lower than threshold and there is no ionization. In the middle figure, the field is slightly larger than threshold causing a streak of ionization. In the right figure, the field is much larger than threshold with a large amount of ionization, but the position density is unrealistically high.

long as possible. For the gas composition it is important that the gas can be effectively ionized by the laser beam, but not by the positron beam. This is because the laser beam will leave neutral gas inside the middle of the hollow plasma channel and the positron beam can ionize this neutral gas.

We estimate that the electric field necessary to ionize atomic hydrogen over a time scale of order $100 \mathrm{fs}$ is approximately $28 \mathrm{GV} / \mathrm{m}$. Simulation results are given in Fig. 2 of the ionization contours in hydrogen for three different beam parameters representing progressively higher field gradients emanating from the bunch. The colors in the figures represent the bunch field intensity with the blue and red regions simply indicating a change in sign with respect to the longitudinal axis. The white ellipse in the center of the figures represents the bunch with all dimensions normalized. Contours plotted in the figures indicate varying degrees of partial ionization in steps of $20 \%$ with the red contour representing $100 \%$ ionization.

In the figure on the left, the field is less than the ionization threshold and there is no ionization. The middle figure is for positron bunch parameters similar to those planned for FACET [11], i.e., $\sigma_{z}=20 \mu \mathrm{m}, \sigma_{r}=10 \mu \mathrm{m}, N=$ $1.8 \times 10^{10}$ corresponding to a maximum field gradient of $\sim 47 \mathrm{GV} / \mathrm{m}$. In this case, some ionization can occur. The figure on the right is for unrealistic beam parameters showing it is possible to completely ionize the gas. Hence, the conclusion is that ionization of the hydrogen by the positron beam is not a serious issue for FACET-like beam conditions. As shown later, hydrogen is also a very good gas to use for achieving laser-induced gas breakdown.

\section{B. Simulations of positron PWFA in hollow plasma channel}

Preliminary OSIRIS [12] modeling of positron PWFA in hollow plasma channels was conducted to verify the selection of the target values and to better quantify the benefits of using a hollow plasma channel. As way of background, it has been demonstrated in experiments that when propagating in uniform plasmas, $e^{+}$beams drive smaller amplitude wakefields than $e^{-}$beams with similar parameters [13]. It was also observed that these positron beams develop a charge halo [14]. As expected, numerical simulations show that the nonlinear plasma focusing force creating the halo also leads to emittance growth [14]. However, simulations also indicate that larger gradients can be driven in hollow plasma channels than in uniform plasmas [6].

We modeled the case of a train consisting of a DB followed by a WB and investigated their propagation in a hollow plasma channel with channel radii from $r_{\mathrm{ch}}=0$ (i.e., uniform plasma, no channel) to $r_{\mathrm{ch}}=2 c / \omega_{p}$. Our idealized plasma channels have circular cross sections with a step-function increase in the plasma density from zero density inside the channel to maximum density in the wall. This maximum density continues for radial distance of at least $2 c / \omega_{p}$.

Simulations focused on the case of two $e^{+}$positron bunches. A DB/WB train can be produced using a masking technique recently demonstrated [14]. The effect of the plasma channel radius on the peak accelerating gradient driven by the $\mathrm{DB}$, as well as on the transformer ratio was calculated. The transformer ratio $R$ is the ratio of the peak accelerating field behind the DB to the peak decelerating field within the DB. A larger accelerating field leads to a shorter plasma length for a given energy gain, while a larger $R$ value leads to a higher energy transfer efficiency from the DB to the WB. For these field studies, the witness bunch is not included in the simulations to avoid the effect of beam loading on the field measurements. The plasma density is either $1 \times 10^{16} \mathrm{~cm}^{3}$ or $5.6 \times 10^{16} \mathrm{~cm}^{-3}$, and the DB is $14 \mu \mathrm{m}$ long with $6.5 \times 10^{9} e^{+}$. The beam is focused at the entrance of the plasma (or channel) to a transverse size of $10 \mu \mathrm{m}$ with an incoming energy of $25 \mathrm{GeV}$ and a normalized emittance of $50 \times 10^{-6} \mathrm{~mm} \mathrm{mrad}$ in the $x$ direction and $5 \times 10^{-6}$ in the $y$ direction. All simulations are performed with a 3D version of the code OSIRIS [12].

Figure 3(a) shows the electron density in the simulation box after $1 \mathrm{~cm}$ of plasma for $r_{\mathrm{ch}}=c / \omega_{p}$. The color contours represent the amount of electron density above the background density. Traveling from left to right, the DB is located at approximately $z=6 c / \omega_{p}$ or $319 \mu \mathrm{m}$ [see Fig. 3(b)]. The WB, which again is not included in the simulation, would be located at approximately $z=3.8 c / \omega_{p}$ or $204 \mu \mathrm{m}$. The density plot shows that the plasma electrons attracted by the $e^{+} \mathrm{DB}$ form an on-axis density spike behind the $\mathrm{DB}$ and in front of the location where the WB would be placed. It also shows that both bunches propagate essentially outside of the plasma, even though the DB (only) loses energy to the wakefield and the WB (only) gains energy from it. This is shown more clearly 

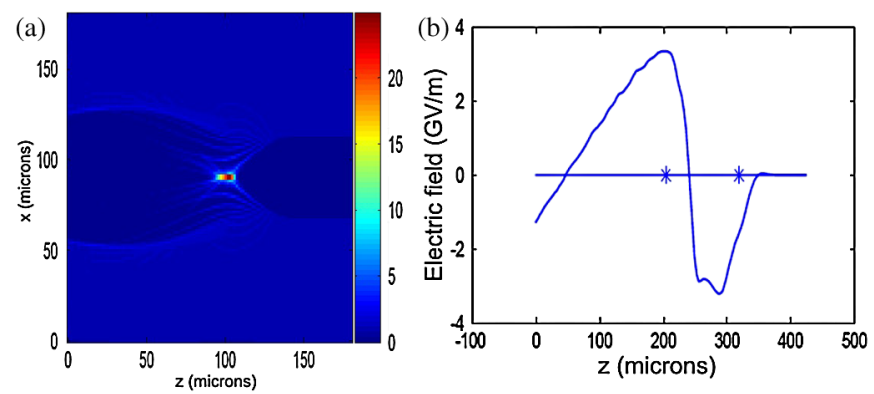

FIG. 3. (a) Electron density within the simulation box after $1 \mathrm{~cm}$ propagation of the drive bunch for $r_{\mathrm{ch}}=c / \omega_{p}$ and $n_{e}=$ $1 \times 10^{16} \mathrm{~cm}^{-3}$. (b) Longitudinal electric field on axis. The asterisks on the $x$ axis indicate the locations of the drive and witness bunches.

in Fig. 3(b), which plots the longitudinal electric field on the beam propagation axis. The asterisks on the axis represent the position of the DB and WB. To achieve maximum energy gain, the WB would be located at the peak positive field point. In this particular case, the peak positive field is slightly larger than the peak negative field yielding a transformer ratio slightly larger than unity. The transformer ratio in a homogeneous plasma (i.e., no hollow channel) is $R=0.5$; therefore, the case in Fig. 3 demonstrates an improvement in transformer ratio by over a factor of 2. This would imply a corresponding increase in the energy transfer efficiency [15].

Operating at higher plasma densities, e.g., $5.6 \times 10^{16} \mathrm{~cm}^{-3}$, can help increase the field strength, in this case by over a factor of 2 compared to the $1 \times 10^{16} \mathrm{~cm}^{-3}$ case, but it also changes the wakefield period. This can complicate the optimum phasing of the WB within the wakefield. Preliminary simulation results indicate that at this higher density, a transformer ratio of $R=1.4$ is possible for $r_{\mathrm{ch}}=2 c / \omega_{p}$, i.e., nearly 3 times better than the uniform plasma case. We should point out that our method for creating the hollow channel permits a wide range of values for $r_{\mathrm{ch}}$ and, hence, achieving $r_{\mathrm{ch}}=2 c / \omega_{p}$ should not be an issue.

\section{DESCRIPTION OF BESSEL-BEAM- GENERATED PLASMA CHANNEL MODEL}

The simulation for predicting the plasma channel formation by an intense Bessel beam is a 1D model requiring solving three mutually coupled component problems. The first deals with the gas/plasma hydrodynamics and solves the conservation equations for mass, momentum, and energy density:

$$
\frac{\partial \xi_{i}}{\partial t}+\nabla \cdot\left(\xi_{i} \mathbf{v}+\varphi_{i}\right)=S_{i}
$$

Mass conservation has

$$
\xi_{1}=\rho, \quad \varphi_{1}=0, \quad \text { and } \quad S_{1}=0,
$$

where $\rho$ is the mass density and $\mathbf{v}$ is the fluid velocity. Momentum conservation has

$$
\xi_{2}=\rho \mathbf{v}, \quad \varphi_{2}=P \cdot \overline{\overline{\mathrm{I}}}, \quad \text { and } \quad S_{2}=0,
$$

where $P$ is the scalar pressure and $\overline{\overline{\mathrm{I}}}$ is the unit tensor. Energy conservation has

$$
\begin{aligned}
& \xi_{3}=\rho \varepsilon+\frac{1}{2} \rho \mathbf{v}^{2}, \quad \varphi_{3}=P \overrightarrow{\mathbf{v}}+\overrightarrow{\mathbf{q}} \\
& S_{3}=\frac{1}{2} \operatorname{Re}(\sigma)\left|\boldsymbol{E}^{2}\right| \pm \text { rate of energy/loss from ionization/recombination, }
\end{aligned}
$$

where $\varepsilon$ is the internal energy per unit mass, $\overrightarrow{\mathbf{q}}$ is the heat flux, $\boldsymbol{E}$ is the laser electric field, and $\sigma$ is the Drude model plasma AC conductivity. The subtractive ionization contribution to the source term $S_{3}$ originates from electron energy loss in collisional excitation/ionization of ions. The additive recombination contribution originates from the carrying away of excess energy during three-body recombination by the third body (the free electron). A Lagrangian "single-fluid" model and an ideal gas equation of state (relating pressure and temperature) are assumed in the hydrodynamics. Spitzer/Braginskii thermal conductivity or flux-limited heat flow is also assumed.

The second component problem is solving for the selfconsistent Bessel beam in the gas target as the plasma develops. If the optical axis is the $z$ axis, the electric field $\boldsymbol{E}$ is given by

$$
\boldsymbol{E}\left(\boldsymbol{r}_{\perp}, z\right)=\hat{\boldsymbol{e}} u\left(\boldsymbol{r}_{\perp}\right) e^{i \beta z},
$$

where $u$ is the amplitude of the field, $\hat{\boldsymbol{e}}$ is the polarization unit vector, and $\beta$ is the wave number parallel to the optical axis. The field amplitude $u$ of the generalized Bessel beam (self-consistent with the plasma) is computed from the Helmholtz equation [8],

$$
\nabla_{\perp}^{2} u\left(r_{\perp}\right)+\kappa^{2} u\left(r_{\perp}\right)=0,
$$

where $\mathbf{r}_{\perp}$ and $\kappa$ are the position coordinate and wave number perpendicular to the optical axis, $\left[\omega^{2} n^{2}(r)\right] / c^{2}=$ $\kappa^{2}+\beta^{2}, \omega$ is the laser frequency, and

$$
n^{2}(\mathbf{r})=\varepsilon_{g}(\mathbf{r})=1+4 \pi \chi_{\text {neutral }}(\mathbf{r})-\frac{\omega_{\mathrm{p}}^{2}(\mathbf{r})}{\omega[\omega+i v(\mathbf{r})]},
$$

where $\varepsilon_{g}$ is the dielectric function of the gas/plasma, $n$ is its refractive index, $\chi_{\text {neutral }}$ is the susceptibility of the neutral component of the gas, $\omega_{p}=\left(4 \pi N_{e} e^{2} / m_{e}\right)^{1 / 2}$ is the plasma frequency $\left(N_{e}\right.$ is the electron density, $e$ is the 


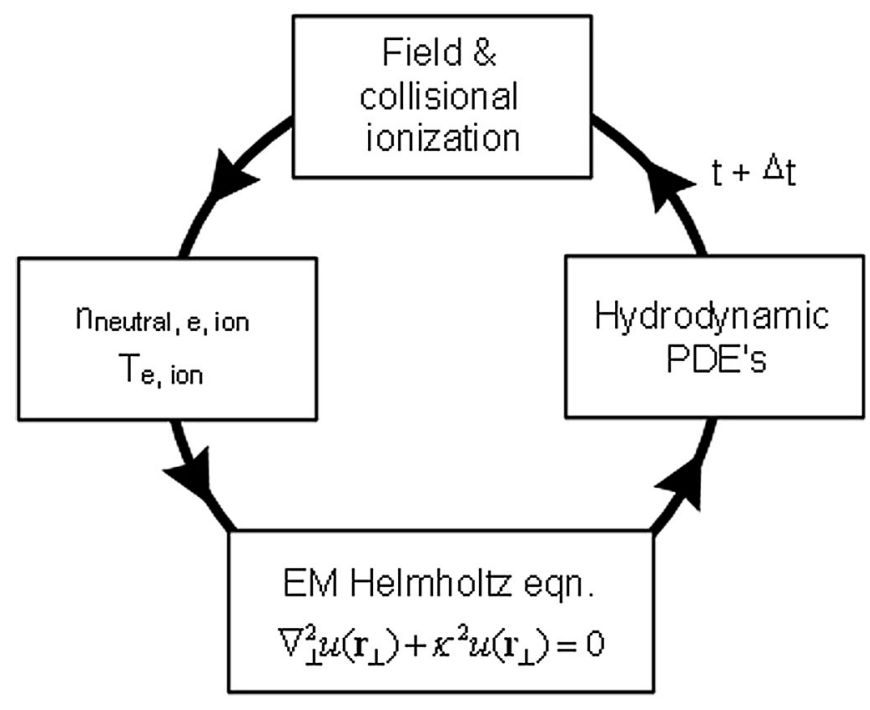

FIG. 4. Flow diagram for Bessel-beam model computations.

electron change, and $m_{e}$ is the electron mass), and $\nu$ is the frequency for electron-neutral and electron-ion collisions. Note that $\chi_{\text {neutral }}, \omega_{p}$, and $\nu$ all depend on the transient state of the plasma as it is ionized and heated by the laser. In vacuum, the solution to Eq. (6) satisfies $u \propto J_{m}(\kappa r)$, where $J_{m}$ is the $m$ th order Bessel function. The index $m$ is imposed by the phase plate in front of the axicon in Fig. 1. For an axicon without a preceding phase plate, $u \propto J_{0}(\kappa r)$. Numerically, Eq. (6) is solved using an adaptive space grid that is interpolated from the Lagrangian hydrodynamics grid at each time step.

The third component problem is solving for the dynamic electron density and ionization state populations through the set of coupled equations,

$$
\begin{aligned}
\frac{d N_{j}}{d t}= & S_{j-1} N_{j-1} N_{e}-\left(S_{j}+\alpha_{j}\right) N_{j} N_{e}+\alpha_{j+1} N_{j+1} N_{e} \\
& +w_{j-1}(|E|) N_{j-1}-w_{j}(|E|) N_{j},
\end{aligned}
$$

where

$$
N_{e}=\sum_{j} Z_{j} N_{j}
$$

is the electron density, $N_{j}$ is the population density of ion state $j, S_{j}$ is the collisional ionization rate from state $j$ to state $j+1, \alpha_{j-1}=a_{j}+b_{j} N_{e}$ is the two-body plus threebody recombination rate from state $j$ to state $j-1$, and $w_{j}$ is the field ionization rate from state $j$ to state $j+1$. For relatively low gas/plasma densities, the excitation and population of excited states within each ion stage can be neglected, especially for the specific case of hydrogen simulated here. For much higher density targets than considered here for our hollow plasma channels, very highly bound states in ions are effectively linked through local thermodynamic equilibrium.

All computations utilize standard finite differencing and are "explicit" in time. The variable time step size, which governs numerical stability, is adjusted at each time step to be smaller than the time scale for the fastest physical process, either heat flow or ionization.

Figure 4 depicts a flow chart of the computational process followed by the model. The iteration begins at an initial state, which typically would be neutral gas at some density and zero electromagnetic field. As the field increases with time, the Helmholtz equation is solved. This then drives the hydrodynamics partial differential equations (PDEs), yielding the temperature and density profiles and allowing, together with the solved $E$ field, the advancement in time of the ionization state through field ionization, and collisional ionization and recombination. This then resets the populations and distributions of the neutrals, electrons, and ions, and their temperatures. The process is repeated by incrementing the time step.

The output of the model includes the time-dependent behavior of a number of parameters including the electron density, velocity of the fluid elements, electron temperature, ion temperature, average ionization, and the self-consistent time and space distribution of the laser electric field.

\section{MODEL RESULTS FOR HOLLOW PLASMA CHANNEL FORMATION}

\section{A. 10-cm hollow plasma channel 1D modeling results}

Table I lists the key parameters used in the model

\begin{tabular}{|c|c|c|}
\hline Parameter & Value & Comments \\
\hline Gas & Hydrogen & $\begin{array}{l}\text { Can be fully ionized by laser beam. Simulations indicate } \\
\text { ionization by positron beam should not be an issue (see text). }\end{array}$ \\
\hline Gas pressure & 0.86 Torr & Yields $n_{e}=6 \times 10^{16} \mathrm{~cm}^{-3}$ when fully ionized. \\
\hline Laser wavelength & $800 \mathrm{~nm}$ & Ti:sapphire laser. \\
\hline Laser pulse length & $60 \mathrm{fs}$ & Typical pulse length for ultrafast Ti:sapphire laser. \\
\hline Bessel beam & $J_{5}$ & Other high-order Bessel beams are sometimes better to use (see text). \\
\hline Input beam radius (HWHM) & $0.7 \mathrm{~cm}$ & $\begin{array}{l}\text { For an axicon approach angle of } 1.8^{\circ} \text {, this is the beam size needed } \\
\text { to create a } 22.3 \mathrm{~cm} \text { long axicon focus length in which } 100 \% \text { ionization } \\
\text { will occur over approximately } 50 \% \text { of its length (see text). }\end{array}$ \\
\hline Axicon approach angle & $1.8^{\circ}$ & See text for discussion on selection of value. \\
\hline
\end{tabular}
to generate a $10-\mathrm{cm}$ long hollow plasma channel using a

TABLE I. Key parameter values for modeling creation of $10-\mathrm{cm}$ long hollow plasma channel. 
5th-order Bessel beam, along with explanatory comments. Expanding upon these comments, there are various tradeoffs when choosing the axicon approach angle. A large angle shortens the axicon focal region and shrinks the radius of the 1 st order lobe of the $J_{5}$ Bessel profile. To achieve an inner channel radius of $22 \mu \mathrm{m}$ requires the peak of the 1st order lobe to be at approximately $27 \mu \mathrm{m}$. An approach angle of $1.8^{\circ}$ produces a $J_{5}$ ring at this radius for $\lambda=800 \mathrm{~nm}$.

With the approach angle selected, the radius of the incoming laser beam determines the length of the axicon focal region. We assume the incoming laser beam will have a Gaussian-like radial intensity distribution. Owing to the weak contributions to the axial intensity distribution from the input beam center and outer edge (see Fig. 9 and the Appendix), only the central portion of the axicon focal region will have sufficient intensity to break down the gas. This means the axicon focal region must be longer than our target length of $10 \mathrm{~cm}$. A half-width-at-half-maximum (HWHM) radius of $0.7 \mathrm{~cm}$ was chosen, which gives an axicon focal length of over $22 \mathrm{~cm}$. As explained next, gas breakdown will occur over approximately $50 \%$ of this length or, in other words, greater than $10 \mathrm{~cm}$.

For the conditions given in Table I, the model indicates that gas breakdown (more specifically, 100\% ionization) occurs when the maximum intensity at the peak of the 1 st lobe is $3.5 \times 10^{14} \mathrm{~W} / \mathrm{cm}^{2}$ and the total laser pulse energy is $45 \mathrm{~mJ}$. However, since it is a $1 \mathrm{D}$ code, the model cannot indicate over what length the breakdown will occur. As shown by Eq. (A17) in the Appendix, if the Bessel-beam focus length is approximately $20 \mathrm{~cm}$, i.e., $z_{\max } \approx 20 \mathrm{~cm}$, and $1-\xi=0.3$, then the breakdown length $L=10 \mathrm{~cm}$. Put another way, within a $10 \mathrm{~cm}$ region centered at the peak intensity point of $3.5 \times 10^{14} \mathrm{~W} / \mathrm{cm}^{2}$, the intensity drops by only $30 \%$, which is still well within the breakdown limits for the gas. (Or, more realistically, one could simply operate at slightly higher pulse energy to ensure breakdown over at least $10 \mathrm{~cm}$.) This means the total needed pulse energy in the $0.7-\mathrm{cm}$ radius input laser beam is $<50 \mathrm{~mJ}$. This corresponds to $<30 \mathrm{~mJ} / \mathrm{cm}^{2}$ fluence, which is well within the damage limits of laser optics. Note that the laser pulse energy required per unit length of channel is entirely determined by the peak intensity required in the lobe for field ionization, and the lobe geometry. At the low hydrogen target densities used here (few $10^{16} \mathrm{~cm}^{-3}$ ), field ionization removes negligible pulse energy per unit length of channel.

Figure 5 shows the model prediction of the radial electron density for the conditions in Table I and assuming $45 \mathrm{~mJ}$ laser pulse energy. These density profiles represent the plasma channel radial distributions at selected times after the peak of the laser pulse corresponding to $t=0$. We see the electron density saturates at $100 \%$ ionization $\left(n_{e}=\right.$ $6 \times 10^{16} \mathrm{~cm}^{-3}$ ) inside the wall of the plasma channel at
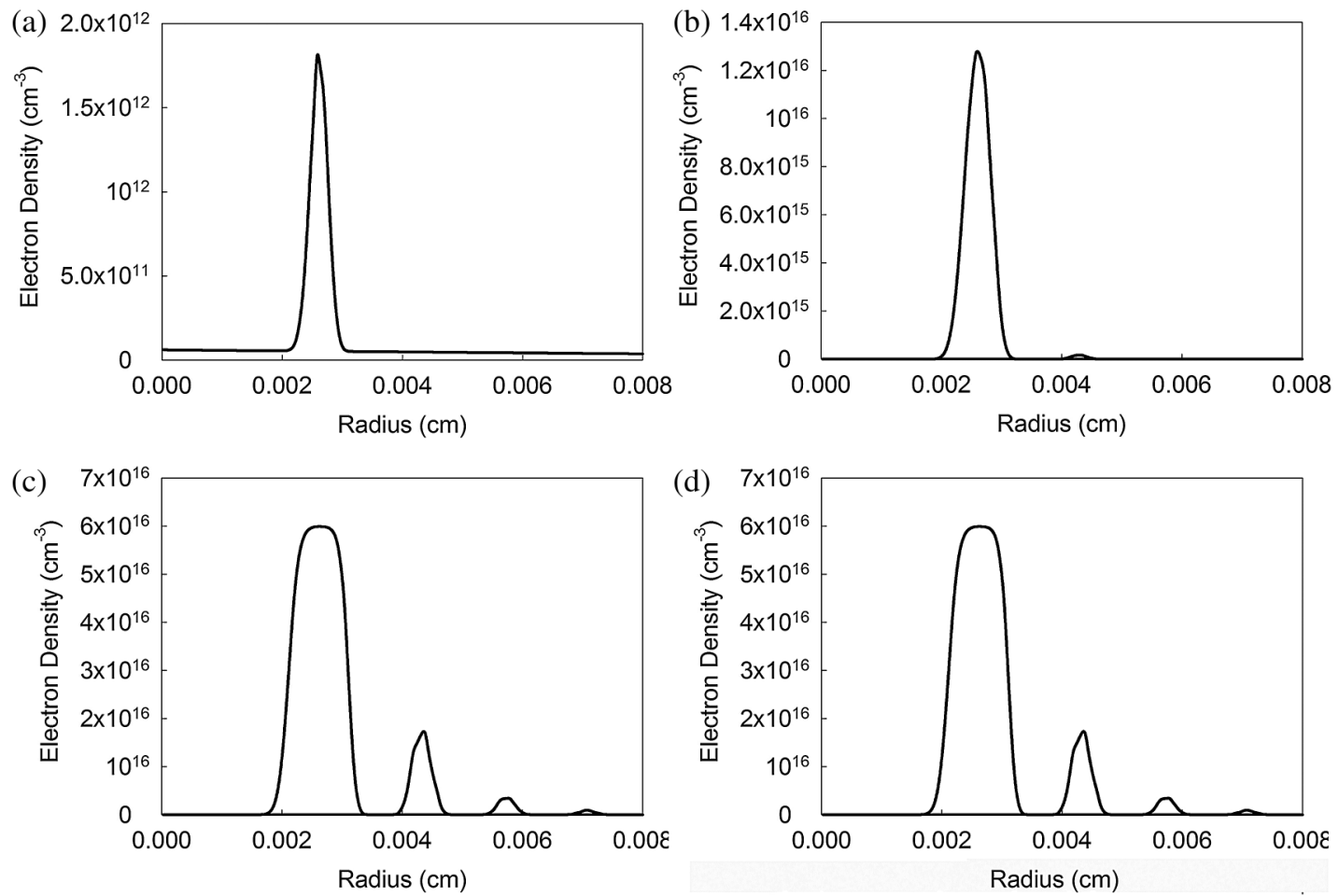

FIG. 5. Model predictions for radial electron density distribution for $45 \mathrm{~mJ}$ pulse energy at different times after the peak of the laser pulse (peak occurs at $t=0$ ). The input laser beam HWHM $=0.7 \mathrm{~cm}$. (a) At 21 fs. (b) At 42 fs. (c) At 63 fs. (d) At 0.27 ps. 

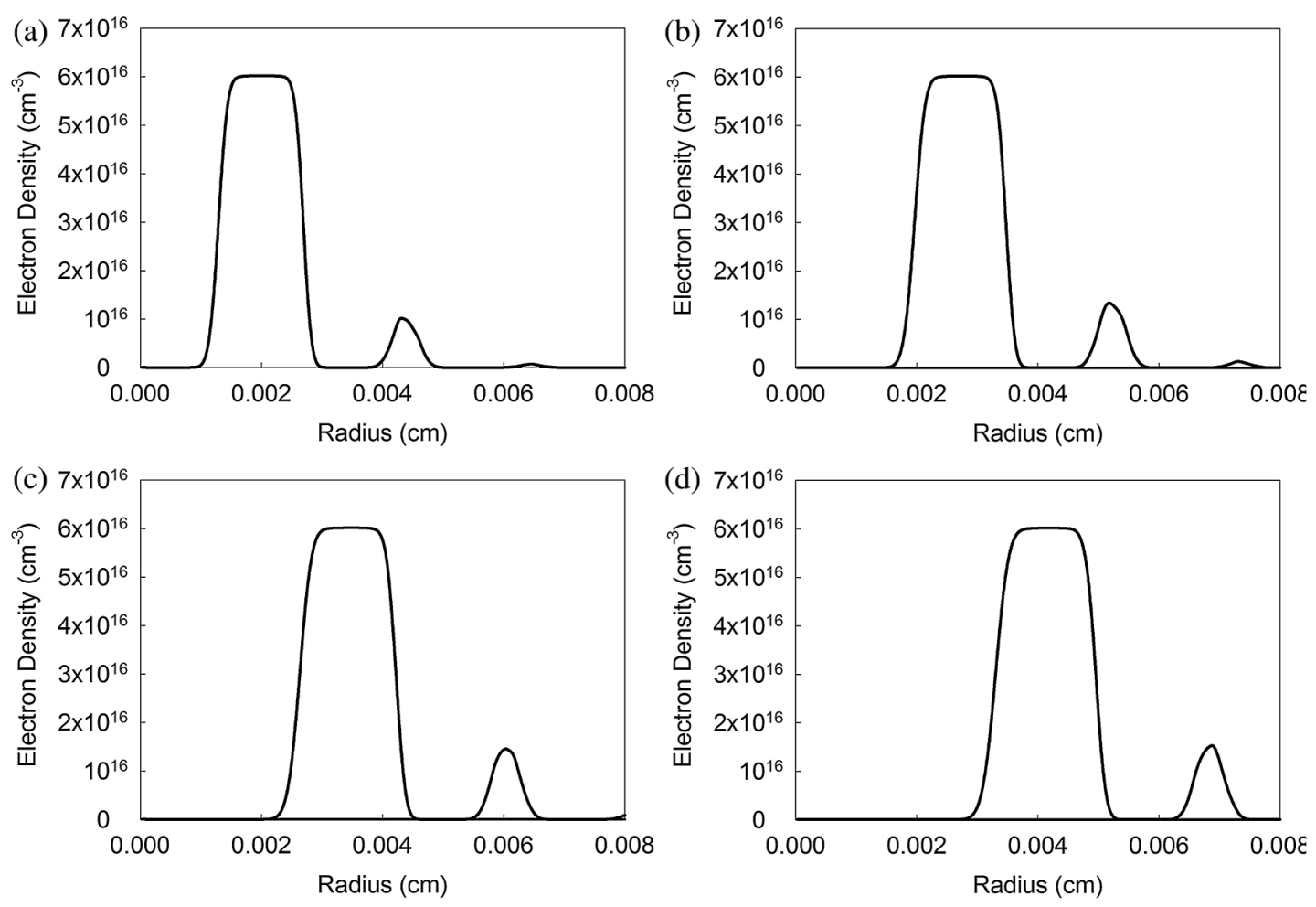

FIG. 6. Electron density results as a function of Bessel-beam order at 102 fs (laser pulse peak occurs at $t=0$ ). (a) $J_{2}$ beam. (b) $J_{3}$ beam. (c) $J_{4}$ beam. (d) $J_{5}$ beam.

$t=63$ fs. It maintains this shape until well after $0.27 \mathrm{ps}$ [see Fig. 5(d)]. Note also that the inner wall radius is at approximately $22 \mu \mathrm{m}$.

\section{B. 100-cm hollow plasma channel 3D modeling results}

Although $10 \mathrm{~cm}$ is a good working length for a proof-ofprinciple demonstration of positron PWFA in a hollow plasma channel, a key question is how do the laser beam requirements scale up with longer lengths? A 100-cm long plasma represents a reasonable scaled-up length where one can imagine connecting these $1-\mathrm{m}$ lengths in series to

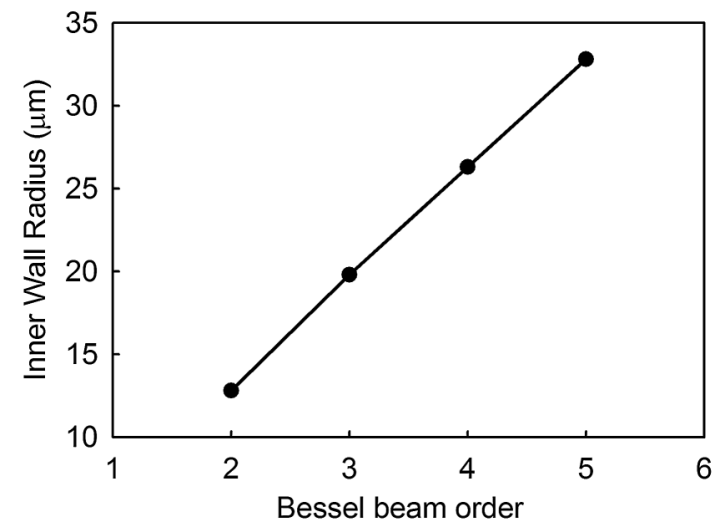

FIG. 7. Dependence of inner wall radius with Bessel-beam order. achieve a hollow plasma channel many meters long. With this in mind, the model was run to determine the parameters needed to achieve a $100 \mathrm{~cm}$ long hollow plasma channel and at the same time generate a 3D plot of the channel shape over the entire length of the channel.

The first set of model runs examined the effect of changing the Bessel-beam order. The laser wavelength, gas pressure, and peak laser intensity at the 1st lobe remained unchanged. To create a longer plasma channel length, the axicon approach angle was reduced to $1.15^{\circ}$ and the input laser beam radius was increased to $2 \mathrm{~cm}$. This

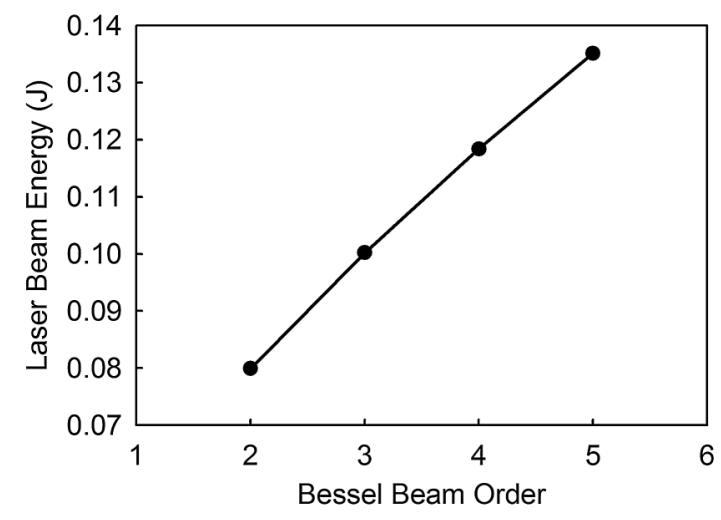

FIG. 8. Dependence of laser pulse energy with Bessel-beam order. 


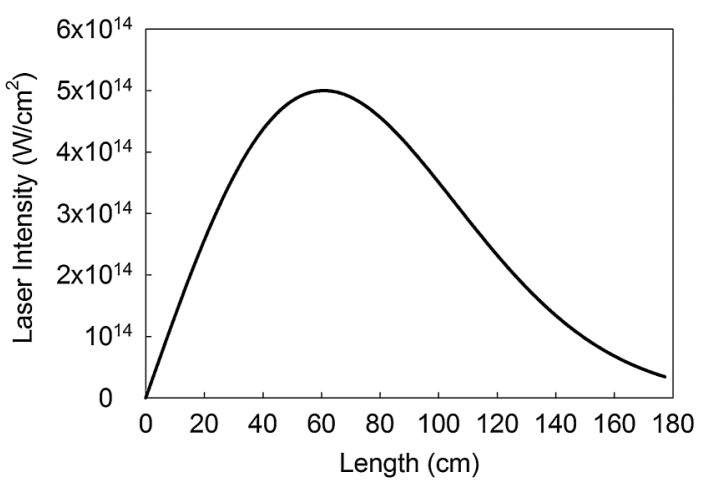

FIG. 9. Plot of the laser intensity as a function of length along the Bessel-beam focus for a peak intensity of $5 \times 10^{14} \mathrm{~W} / \mathrm{cm}^{2}$.

(a)
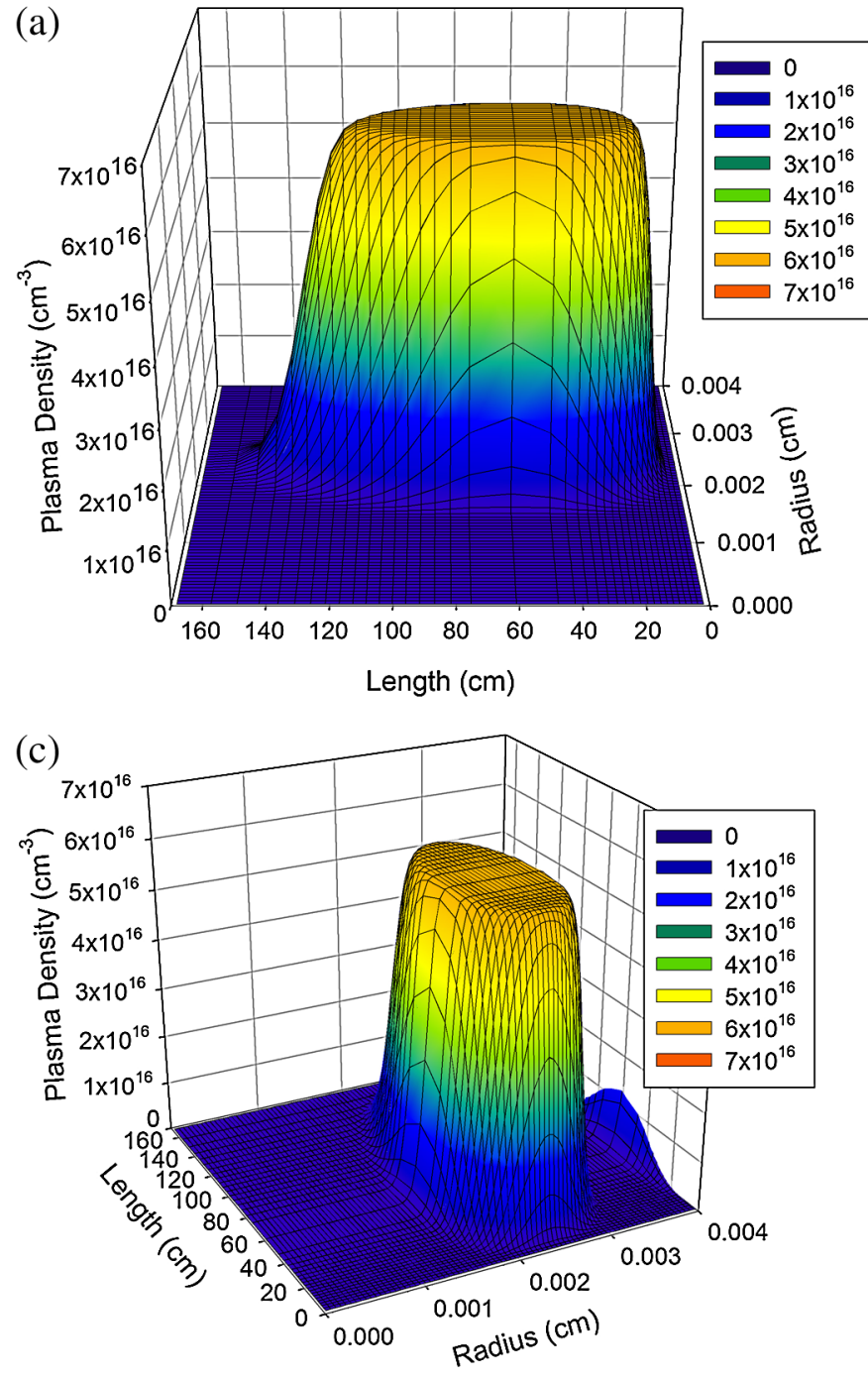

resulted in an axicon focal length of $100 \mathrm{~cm}$. (Achieving gas breakdown over the entire $100 \mathrm{~cm}$ length was not of concern at this point in the analysis.)

Figure 6 gives the model results for the electron radial density profiles after the channel has fully formed at $t=102 \mathrm{fs}$ using laser beams with different higher-order Bessel profiles. We see that in all cases the target electron density is reached $\left(6 \times 10^{16} \mathrm{~cm}^{-3}\right)$ with a plateau indicating $100 \%$ ionization within the wall. The inner radius position of the channel wall increases as the Bessel-beam order increases. A plot showing how the wall radius varies is given in Fig. 7. This indicates achieving our target radius of 22 microns would be possible using a $J_{4}$ Bessel beam.

As the Bessel-beam order increases, the laser pulse energy required to cause gas breakdown also increases. This is plotted in Fig. 8. This implies that achieving a
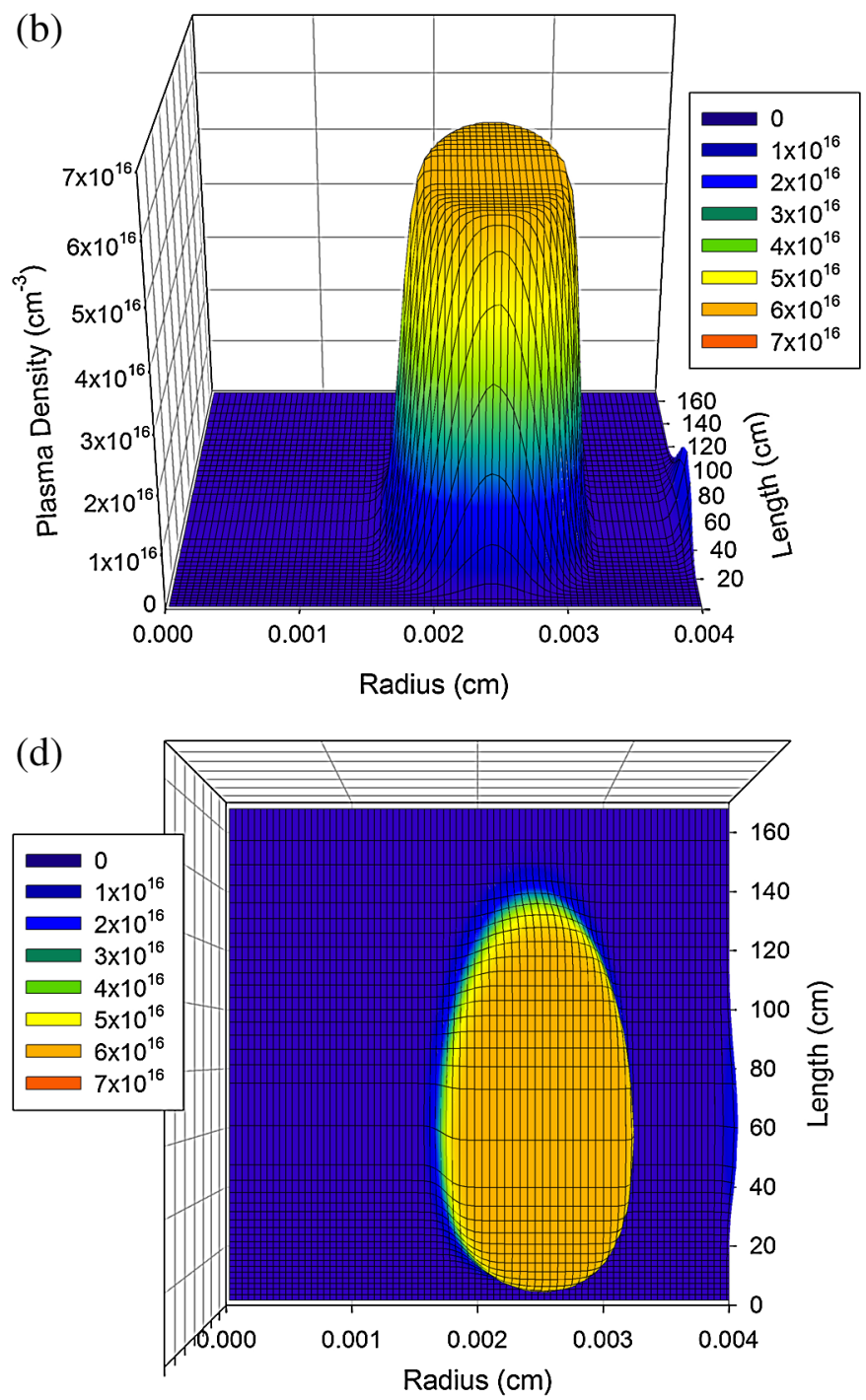

FIG. 10. 3D plots of plasma density distribution for the case of a peak laser intensity of $5 \times 10^{14} \mathrm{~W} / \mathrm{cm}^{2}$. The input laser beam $\mathrm{HWHM}=2 \mathrm{~cm}$. (a) Showing length of plasma channel. (b) Showing width of plasma channel. (c) Showing 3D perspective. (d) Showing length and width of channel. 
$22-\mu \mathrm{m}$ radius using a $J_{4}$ Bessel beam would need about $120 \mathrm{~mJ}$ pulse energy.

Next, a smaller approach angle of $1.6^{\circ}$ was chosen rather than $1.8^{\circ}$ for the $10 \mathrm{~cm}$ case. As a consequence, the runs also assumed a higher peak laser intensity of $5 \times 10^{14} \mathrm{~W} / \mathrm{cm}^{2}$. The higher intensity helps compensate for the smaller angle, which tends to lower the intensity within the Bessel-beam focal region. The runs also used a shorter laser pulse length of $40 \mathrm{fs}$, which is easily obtainable with existing lasers.

A 3D plot was created by forming a composite of 1D runs. This is a reasonable approach because (1) within their depth of focus, Bessel beams are invariant in form along the propagation axis [16], and (2) axial variation of plasma conditions is much weaker than the radial variation. The model was modified to produce a file that plots the intensity as a function of length for a particular value of the peak axial laser intensity. As before, it assumes a Gaussian input intensity profile for the laser beam incident on the axicon. The model run for the $5 \times 10^{14} \mathrm{~W} / \mathrm{cm}^{2}$ case yielded the axial intensity distribution plotted in Fig. 9. As would be expected for a Gaussian input beam, the intensity increases until it reaches a peak value of $5 \times 10^{14} \mathrm{~W} / \mathrm{cm}^{2}$ at approximately $61 \mathrm{~cm}$ and then gradually decreases towards the right of the plot at $180 \mathrm{~cm}$.

To calculate the electron density over the full focal length of the Bessel beam, the code performed a large sequence of runs corresponding to the peak intensity variation along the focal length as seen in Fig. 9. Then, to construct a 3D plot of the electron distribution, the radial density distributions generated by the discrete set of peak intensities from the range plotted in Fig. 9 are concatenated. Figure 10 gives the results of performing this procedure. Shown is the 3D electron density plot of the plasma channel from various perspectives. Figures 10(a) and 10(d) indicate that the plateau of the electron density, i.e., region of $100 \%$ ionization, is well over $100 \mathrm{~cm}$ long. Figure 10(d) also shows that the inner radius of the channel stays well within our target value of $\sim 20 \mu \mathrm{m}$ over much of the $100 \mathrm{~cm}$ length.

The field ionization rate [17] is exponential $[\sim \exp (-\eta / E)]$ in the laser electric field strength $E$, where $\eta$ depends on the ion and state. This causes the electron density to increase fairly rapidly from $z=0$ to its fully ionized value at $z=24 \mathrm{~cm}$. It then maintains this fully ionized value until about $z=104 \mathrm{~cm}$. After this point it decreases at a rate slower than at the beginning of the channel. This is because the intensity increases faster ( $\sim$ linearly) with $z$ at the beginning of the focus (near $z=0$ ) than at the end (see Fig. 9). As explained in the Appendix, the curve in Fig. 9 is described by $I(z) \sim z \exp \left\{-\ln 2\left[\rho(z) / \rho_{1 / 2}\right]^{2}\right\}$, where $\rho(z)$ is the mapping from the input beam radius to positions along $z$ and $\rho_{1 / 2}$ is the input beam half-width-at-half-maximum (HWHM).

\section{DISCUSSION AND SUMMARY}

In summary, the model predicts a $10-\mathrm{cm}$ long hollow plasma channel with inner channel radius $r_{\text {ch }}=22 \mu \mathrm{m}$ and plasma density $n_{e}=6 \times 10^{16} \mathrm{~cm}^{-3}$ can be created in 0.86 Torr of hydrogen gas using a Ti:sapphire laser $(\lambda=800 \mathrm{~nm})$ with pulse length $=60 \mathrm{fs}$, pulse energy $<50 \mathrm{~mJ}$, and laser beam radius of $0.7 \mathrm{~cm}$. Preliminary positron PWFA simulations predict this channel radius and plasma density should enable transformer ratios $>1$. The model also predicts a $100-\mathrm{cm}$ long hollow channel can be formed using $122 \mathrm{~mJ}$ pulse energy in a 40-fs pulse with a beam radius of $2 \mathrm{~cm}$. It should be pointed out that Ti: sapphire laser systems, based upon commercial components, are available that produce hundreds of $\mathrm{mJ}$ output pulses with pulse lengths as short as $\sim 25 \mathrm{fs}$ ranging to $>100$ fs.

Optical damage should not be an issue because of the favorable scaling when using axicon optics, i.e., longer plasma channels require larger diameter laser beams on the axicon and kinoform. Hence, the fluence levels should be well within the damage threshold for these optics. Potential problems with optical nonlinearities when dealing with ultrashort laser pulses can be avoided by using reflective optics wherever possible, including the axicon. Fortunately, the phase plate can be made very thin, thereby minimizing nonlinear distortion of the ultrashort pulses.

\section{ACKNOWLEDGMENTS}

This work was supported by the U.S. Department of Energy, Grants No. DE-FG02-08ER85040 and No. DEFG02-97ER41039.

\section{APPENDIX: FIRST-ORDER ANALYSIS OF INTENSITY DISTRIBUTION ALONG AXICON FOCAL REGION}

Referring to Fig. 11, from Eq. (6) of Ref. [18], the mapping of the transverse intensity distribution along the axicon focal region is given by

$$
z(\rho)=c_{\alpha} \rho
$$

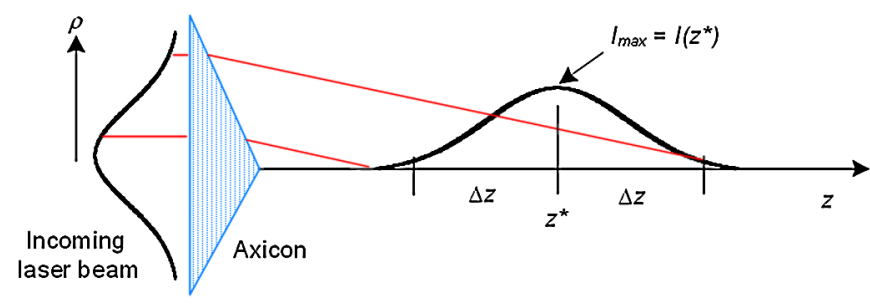

FIG. 11. Mapping of Gaussian-shaped incoming laser beam onto axicon focal axis. 
where $c_{\alpha}=(1 / \tan \gamma)-\tan \alpha, \gamma$ is the approach angle and $\alpha$ is the axicon base angle. From Eq. (9) of Ref. [18], it follows that the intensity distribution is given by

$$
I(z)=\frac{\pi k \sin \gamma}{2} \frac{z}{c_{\alpha}} I_{\text {in }}[\rho(z)] \frac{(1+\cos \gamma)^{2}}{4},
$$

where the peak of the intensity distribution occurs at $z=z^{*}$ (see Fig. 11). We assume the intensity drops off symmetrically from the peak at $z=z^{*} \pm \Delta z$, so that from Eq. (A2) we can write

$$
\frac{I\left(z^{*} \pm \Delta z\right)}{I\left(z^{*}\right)}=\frac{\left(z^{*} \pm \Delta z\right)}{z^{*}} \frac{I_{\text {in }}\left[\rho\left(z^{*} \pm \Delta z\right)\right]}{I_{\text {in }}\left[\rho\left(z^{*}\right)\right]} .
$$
i.e.,

If we assume a Gaussian input for the intensity profile,

$$
I_{\text {in }} \propto \exp \left(-\rho^{2} / \rho_{0}^{2}\right),
$$

where $\rho_{0}$ characterizes the width of the laser beam, then

$$
\frac{I\left(z^{*} \pm \Delta z\right)}{I\left(z^{*}\right)}=\left(1 \pm \frac{\Delta z}{z^{*}}\right) \exp \left[\frac{-z^{*^{2}}}{c_{\alpha}^{2} \rho_{0}^{2}}\left(\frac{(\Delta z)^{2}}{z^{*^{2}}} \pm \frac{2 \Delta z}{z^{*}}\right)\right] .
$$

It is convenient to rewrite Eq. (A2) as

$$
I(z)=A z I_{\text {in }}[\rho(z)],
$$

where all the multiplicative factors have been lumped into the constant $A$. The derivative with respect to $z$ for the intensity function is then

$$
\frac{d I}{d z}=A I_{\text {in }}[\rho(z)]+A z \frac{d I_{\text {in }}}{d \rho} \frac{d \rho}{d z} \text { and } \frac{d \rho}{d z}=\frac{1}{c_{\alpha}} .
$$

Hence, the maximum intensity location along the axicon focus satisfies the condition $d I / d z=0$, which implies from Eq. (A7) that

$$
I_{\text {in }}[\rho(z)]=z \frac{d I_{\text {in }}}{d \rho} \frac{d \rho}{d z} .
$$

Substituting the value for $I_{\text {in }}$ from Eq. (A4), we find that

$$
\frac{d I_{\text {in }}}{d \rho} \propto \frac{-2 \rho}{\rho_{o}^{2}} \exp \left(-\rho^{2} / \rho_{o}^{2}\right) .
$$

From this it can be shown that the axial location of maximum intensity is when

$$
z^{*}=\frac{c_{\alpha}}{\sqrt{2}} \rho_{0}
$$

If the Gaussian input beam half-width-at-half-maximum (HWHM) is $\rho_{1 / 2}$, then

$$
\exp \left(\frac{-\rho_{1 / 2}^{2}}{\rho_{0}^{2}}\right)=\frac{1}{2}
$$

Solving for $\rho_{0}$ and substituting into Eq. (A10) we find

$$
z^{*}=\frac{c_{\alpha}}{\sqrt{2}} \frac{\rho_{1 / 2}}{(\ln 2)^{1 / 2}} .
$$

But, from Eq. (A1), $z_{\max }=c_{\alpha} \rho_{\max }$, from which it can be concluded that $z^{*} \approx z_{\max } / 2$. Next, let

$$
\xi=\frac{I\left(z^{*} \pm \Delta z\right)}{I\left(z^{*}\right)}=(1 \pm x) \exp \left[\frac{-z^{*^{2}}}{c_{\alpha}^{2} \rho_{0}^{2}}\left(x^{2} \pm 2 x\right)\right]
$$

where $x=\Delta z / z^{*}$. The parameter $\xi$ represents the fractional decrease in intensity. For $x \ll 1$,

$$
\xi \approx(1 \pm x)\left[1-\frac{1}{2} x(x \pm 2)\right]
$$

which means that

$$
x=\sqrt{\frac{2}{3}}(1-\xi)^{1 / 2} .
$$

Therefore, since $x=\Delta z / z^{*}$, we find that $L$, defined as the length over which the intensity deviates from the peak intensity within a fraction of $1-\xi$, is given by

$$
L=2 \Delta z=2 z^{*} \sqrt{\frac{2}{3}}(1-\xi)^{1 / 2} \cong z_{\max } \sqrt{\frac{2}{3}}(1-\xi)^{1 / 2} .
$$

As an example, if $1-\xi=0.3$, corresponding to a $30 \%$ drop from the peak intensity at $\pm \Delta z$, then

$$
z_{\max } \cong \frac{L}{0.45} \approx 2 L \text {. }
$$

Therefore, to first order, if we wish to have $L=10 \mathrm{~cm}$ with only a $30 \%$ drop in intensity at $\pm \Delta z$, then $z_{\max }$ needs to be approximately $20 \mathrm{~cm}$.

[1] P. Chen, J.M Dawson, R. W. Huff, and T. Katsouleas, Phys. Rev. Lett. 54, 693 (1985).

[2] I. Blumenfeld et al., Nature (London) 445, 741 (2007).

[3] S. Lee et al., Phys. Rev. ST Accel. Beams 5, 121301 (2002).

[4] J. B. Rosenzweig, Phys. Rev. Lett. 58, 555 (1987).

[5] C.E. Clayton et al., Phys. Rev. Lett. 88, 154801 (2002).

[6] S. Lee, T. Katsouleas, R. G. Hemker, E. S. Dodd, and W. B. Mori, Phys. Rev. E 64, 045501 (2001).

[7] K. A. Marsh et al., in Proceedings of the 20th Particle Accelerator Conference, Portland, OR, 2003 (IEEE, New York, 2003), p. 731, http://accelconf.web.cern.ch/ AccelConf/p03/PAPERS/FOAB011.PDF.

[8] J. Fan, E. Parra, I. Alexeev, K. Y. Kim, H. M. Milchberg, L. Ya. Margolin, and L. N. Pyatnitskii, Phys. Rev. E 62, R7603 (2000).

[9] N. E. Andreev, Yu. A. Aristov, L. Ya. Polonskii, and L. N. Pyatnitskii, Sov. Phys. JETP 73, 969 (1991).

[10] N.E. Andreev, S. S. Bychkov, V. V. Kotlyar, L. Ya. Margolin, L. N. Pyatnitskii, and P.G. Serafimovich, Quantum Electron. 26, 126 (1996). 
[11] M. J. Hogan et al., New J. Phys. 12, 055030 (2010).

[12] R. G. Hemker et al., in Proceedings of the 18th Particle Accelerator Conference, New York, 1999 (IEEE, New York, 1999), p. 3672, http://accelconf.web.cern.ch/ AccelConf/p99/PAPERS/FRA124.PDF.

[13] B.E. Blue et al., Phys. Rev. Lett. 90, 214801 (2003).

[14] P. Muggli et al., Phys. Rev. Lett. 101, 054801 (2008).
[15] M. Zhou, Master thesis, UCLA, 2005; Ph.D. dissertation, UCLA, 2008.

[16] J. Durnin, J. J. Miceli, Jr., and J. H. Eberly, Phys. Rev. Lett. 58, 1499 (1987).

[17] M. V. Ammosov, N. B. Delone, and V.P. Krainov, Zh. Eksp. Teor. Fiz. 91, 2008 (1986) [Sov. Phys. JETP 64, 1191 (1987)].

[18] C. G. Durfee, III, J. Lynch, and H. M. Milchberg, Phys. Rev. E 51, 2368 (1995). 